\title{
Scaling of dot numerosity
}

\author{
TAROW INDOW and MASASHI IDA \\ Keio University, Tokyo, Japan
}

\begin{abstract}
Subjective impression of numerosity of dots randomly scattered in an area of $19.5 \times 24.5 \mathrm{~cm}$, $36 \sim 1,010$ in number $(\phi)$, was scaled by four different methods with five subjects. The absolute identification showed that the number of dots is underestimated if $\phi>10$. Each of two scales based upon ratio judgments is a power function of $\phi$ with the exponent of 0.87 , and each of two scales based upon difference judgments is also a power function of $\phi$ when the origin of the scale is appropriately defined. The relationship between the four scales and comparison with scales in other sense modalities were discussed from the viewpoint of scaling methodology.
\end{abstract}

A glimpse of a collection of things, such as beans, trees, or a crowd of people (Mann, 1970), will give us the impression of numerousness (S. S. Stevens, 1939; Taves, 1941) or numerosity (Klahr, 1973; Krueger, 1972). If there are two such collections, not only the human but also animals will be able to tell which collection is greater, provided the two are not too close in number. Thus, perceiving numerosity may be said to be as elementary as perceiving brightness, etc. In one respect, the response of adults to numerosity has a special feature. They can name the number of elements in a given collection. In other words, stimulus and response are specified in the same term. Of course, it does not imply that the subjective number should coincide with the objective number of elements. The number of participants in a political demonstration estimated by the organizing committee is often twice as large of the number reported by the police authorities.

The present study consisted of four experiments in which four different methods of scaling were applied to dots randomly scattered in a plane. The number of dots was too large for counting, but not so large that each dot lost its individuality and distinctiveness. A scale of numerosity may not be as useful in practice as scales of brightness (e.g., Hopkinson, 1957; J. C. Stevens \& S. S. Stevens, 1963) and of loudness (e.g., Garner, 1954; S. S. Stevens, 1955, Note 1). However, compared with other intensity attributes, numerosity will be of interest from the viewpoint of scaling methodology. The reason will be stated in the last section.

Let $\phi$ represent a stimulus value, the objective number of dots, and $\psi_{x}(\phi)$ the scale values assigned to $\phi$ by an experimental method $x$, where $x$ varies from I to IV in the present study. The numerosity of dots may be varied in two ways. One will be to change $\phi$, the number of dots, keeping constant the size of the area in which dots are scattered. In this case, the density of dot pattern concomitantly increases with $\phi$. The other way will be to change $\phi$ but to keep constant the average interdot interval. In this case, the size of dot pattern increases with $\phi$. The first method was used in the present study and $\phi$ represents the number of dots in an area of $19.5 \times$ $24.5 \mathrm{~cm}$. Both aspects, numerosity and density, were confounded and, without going into the question of which aspect is the actual counterpart of the scales $\psi_{\mathrm{x}}(\phi)$, the term numerosity will be used in the sections to follow.

\section{EXPERIMENTATION}

\section{Subjects}

Five volunteers participated as subjects in all four experiments. Four (Y.A., Y.I., H.K., and R.S.) were undergraduate students in the Department of Psychology and one (T.I.) was the senior author. Concerning numerosity, all were equally naive, but T.I. had had extensive experience in serving as a subject in this type of experiment with various sense modalities. The junior author worked as the experimenter.

\section{Stimuli}

A number of random dot patterns were provided. Each pattern consisted of $\mathrm{m}$ black circles, $3 \mathrm{~mm}$ in diameter, pasted on a sheet of transparent vinyl chloride, $30 \mathrm{~cm}$ long, $25 \mathrm{~cm}$ high, and $0.125 \mathrm{~mm}$ thick. On a sheet, dots were arranged randomly within an area of $19.5 \times 24.5 \mathrm{~cm}$, with the constraint that none of dots overlap among sheets that were to be overlaid. Thus, it was possible, by combining appropriate sheets, to generate a stimulus consisting of dots of almost any number, $\phi$, ranging, if necessary, from 2 to 1,200 . Usually, a stimulus was composed of two to four vinyl sheets which were tightly overlaid on a glossy white board. Dots on the furthest back sheet appeared slightly different in color from those on the front sheet. This slight heterogeneity in color of dots in a pattern was not very disturbing to the subject, however. For a value of $\phi$, a number of different dot patterns were randomly generated.

\section{Presentation}

Up to four dot patterns could be presented simultaneously in a row on the table. Each was almost perpendicular to the line of sight of the subject but slightly tilted so as to avoid surface reflection of the illuminating light from above. As the viewing distance was approximately $1.2 \mathrm{~m}$, a dot pattern subtended about $11^{\circ} \times 9^{\circ}$, and when four dot patterns were presented, the spread was over a subtense of $64^{\circ}$. In front of the subject was a shutter that was operated by the subject according to the 
signal given by the experiment. When opened, one or more stimuli were presented to the subject; during the interval between presentations, the subject was exposed to the white surface of the shutter. The observation was always made binocularly, and when more than one stimulus were presented, the subject was encouraged to scan freely from one stimulus to another. In all experiments, the subject was urged to respond quickly but was given as much time to respond as the subject desired. As soon as the subject responded, the shutter was closed and a new presentation was given after an appropriate interval.

\section{Experiments}

The study consists of four experiments. The stimulus display 'and the task in each experiment are schematically given in parenthesis where alphabetical letter(s) represent(s) dot pattern(s), and the bar above a letter indicates that $\phi$ of the dot pattern is to be adjusted by the subject.

Experiment I: Absolute identification of 11 stimuli presented one at a time. [A: How many dots in A?]

Experiment II: Ratio estimation in pairwise presentation of the same 11 stimuli. [A, B: What is the ratio, B to A?]

Experiment III: Extrapolation in which matching three adjacent intervals was continued successively. $[A, B, \bar{C}, \bar{D}$, or $\bar{A}$, $\bar{B}, C, D$ : Adjust $C$ and $D$ or $A$ and $B$ so that $(B-A)=$ $(C-B)=(D-C)$ in impression.]

Experiment IV: Extrapolation in which summation or subtraction by a given amount was continued successively. [A, B, $\bar{C}$ : Adjust $C$ so that $(B+A)=C$ or $(B-A)=C$ in impression.]

As shown in Table 1, the experiments were carried out individually over six sessions, with some sessions lasting $\mathbf{4 0} \mathrm{min}$ and some about $2 \mathrm{~h}$. The schedule was the same for the five subjects. For example, each subject repeated Experiment I six times and Experiment IV twice, and so forth.

\section{RESULTS}

The results of each experiment are first analyzed separately for each subject and then synthesized to give the representative picture. Finally, the results of all the experiments, I to IV, are compared and displayed in a single figure (Figure 11).

\section{Experiment I: Absolute Identification}

A set of 11 dot patterns, $\phi_{\mathrm{i}}$, were used, where $\phi$ ranged from 36 to 1,010 with approximately uniform steps on a logarithmic scale. The stimuli were presented one by one with an interval of about $5 \mathrm{sec}$. Presentation order was chosen randomly, with the constraint that neither $\phi_{1}$ nor $\phi_{11}$ come as the first stimulus. Each $\phi_{i}$ was presented only once in a session. The subject was instructed to name as quickly as possible the number of dots in $\phi_{i}$ without counting. Such naming involved transforming the subject's impression of numerosity into a value on his own subjective scale of number. For a value of $\phi_{i}$, dot pattern was randomly changed over sessions and over subjects.

Figure 1 shows two examples of individual data. Each point in the individual data represents one estimation: the subjective number of dots. Hence, the ordinate, $\dot{\psi}_{\mathrm{I}}(\phi)$, is absolute in the sense that no transformation is admissible to the unit as well as to
Table 1

Schedule of Experiments (I to IV) Over Six Sessions

\begin{tabular}{|c|c|c|c|c|c|c|}
\hline \multirow{2}{*}{$\begin{array}{l}\text { Order } \\
\text { Within } \\
\text { Session }\end{array}$} & \multicolumn{6}{|c|}{ Session } \\
\hline & 1 & 2 & 3 & 4 & 5 & 6 \\
\hline 1 & I & I & I & IV & I & I \\
\hline 2 & II & II & II & I & II & I \\
\hline 3 & III & III & IV & II & III & III \\
\hline
\end{tabular}

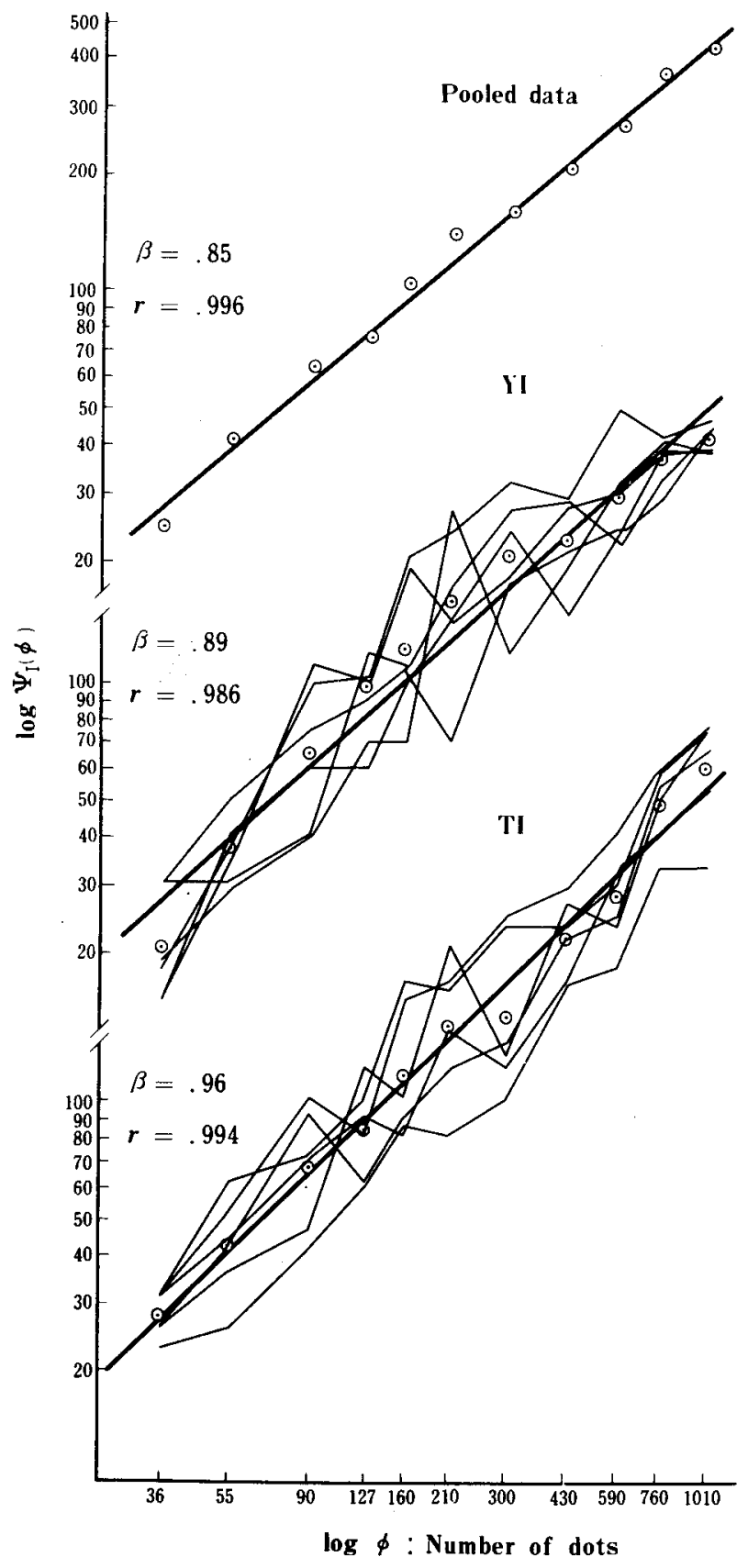

Figure 1. The scales $\psi_{1}$ obtained in Experiment I as functions of $\phi$ in log-log coordinates. 
the origin. No matter how the subject interprets, for example, the number of 100 on his subjective scale of number, a response " 100 " given to a stimulus implies that there are 100 dots as the subject interprets it.

I.1. When plotted in $\log -\log$ coordinates, the relation between the subjective number of dots, $\psi_{\mathrm{I}}$, and the objective number, $\phi$, was approximately linear and hence fitted by a power function:

$$
\psi_{\mathrm{I}}(\phi)=\alpha \phi^{\beta} .
$$

1.2. Scatter of points for a given $\phi_{i}$ is due to three factors: systematic shifts between sessions of the slope, $\beta$, and of the intercept, $\log \alpha$, in addition to random errors. By the nature of the task, the results of Experiment I was supposed to be most susceptible to the experience of the subjects in the remaining experiments, especially Experiments III and IV. Hence, Experiment I was carried out first in each, except in the fourth, session (Table 1). It was not possible, however, to discern any remarkable difference in $\psi_{\mathrm{I}}$ of the first session, where Experiment I was performed prior to all other experiments, and also in $\psi_{\mathrm{I}}$ of the fourth session, where Experiment I was performed right after Experiment IV. In the individual examples in the lower part of Figure 1, six sets of $\psi_{I}$ are given in addition to representative values. In an example plotted at the bottom (TI), the six curves are relatively parallel and uncrossed, which indicates that the between-session fluctuation is mainly due to the shift of $\log \alpha$. On the other hand, the shift of $\beta$ is predominant in the other example (YI) plotted in the middle. The shift of $\log \alpha$ takes place when the modulus for transforming numerosity into the subjective scale of number fluctuates from session to session. The shift of $\beta$ is more complex in nature, and fluctuation of the subjective scale of number may be involved. The geometric mean of these six responses for $\phi_{i}$ is shown by a circle in these plots. Each circle at the top represents the average of the geometric means of the four subjects, except R.S. (see I.5.).

1.3. Equation 1 was fitted by the method of least squares with equal weights to all the plotted points.
The results are given in Figure 1 as well as in Table 2 . As an index of the goodness of fit of Equation 1, the correlation coefficient $r$ between values shown by circles in Figure 1 and $\log \phi_{i}$ is used (Table 2). Notice that $\beta<1$ in all the cases.

I.4. The value $\bar{\phi}$ in Table 2 represents the numerosity at which the subjective number coincides with the objective one, $\psi_{I}(\bar{\phi})=\bar{\phi}$, and $\bar{\phi}$ was obtained by extrapolation:

$$
\log \bar{\phi}=(\log \alpha) /(1-\beta) .
$$

As $\beta<1$, overestimation $\psi_{\mathrm{I}}(\phi)>\phi$ takes place for $\phi<\bar{\phi}$ and underestimation $\psi(\phi)<\phi$ for $\phi>\bar{\phi}$. In the case of T.I., $\psi_{\mathrm{I}}(\phi)<\phi$ throughout $\phi>0$ and $\Phi(>0)$ did not exist.

I.5. After all the experiments were completed, it was found that one subject (R.S.) adopted a particular strategy in responding that was somewhat against the instructions. Whenever a dot pattern was presented, he paid attention to a small section first and roughly estimated the number of dots within that section. Then he estimated how many of these sections were contained in the whole pattern, and then, by multiplication, estimated the total number of dots. In the last row of Table 2, the results, with his data excluded, are given; the results at the top of Figure 1 represent this case.

1.6. In Experiment I, the response latencies were also measured. The medians, $t$, of the respective subjects are plotted against $\log \phi_{\mathrm{i}}$ in Figure 2 . Clearly, the subjects were divided into two types. For three subjects, $t$ was at the level of $2 \sim 4 \mathrm{sec}$ and increased only slightly as a function of $\phi$, which would imply that the naming of number did not involve counting of dots. For two subjects, the increase of $t$ was much larger and $t$ was at a higher level from the beginning. The subject mentioned in I.5., R.S., belonged in the latter group. From his verbal report, it was clear that the other subject in this group (Y.A.) neither used the strategy of R.S. nor depended upon counting.

\section{Experiment II: Ratio Estimation}

A pair of stimuli, $\phi_{\mathrm{j}}$ and $\phi_{\mathrm{k}}$, were presented, and

Table 2

\begin{tabular}{|c|c|c|c|c|c|c|c|c|c|c|c|c|}
\hline \multirow[b]{2}{*}{$\mathrm{S}$} & \multicolumn{4}{|c|}{ Experiment I } & \multicolumn{2}{|c|}{ Experiment II } & \multicolumn{3}{|c|}{ Experiment III } & \multicolumn{3}{|c|}{ Experiment IV } \\
\hline & $\beta$ & $\log \alpha$ & $\bar{\Phi}$ & $\mathbf{r}$ & $\beta$ & $\mathbf{r}$ & c & $\beta$ & $\mathrm{r}$ & c & $\beta$ & $\mathrm{r}$ \\
\hline $\begin{array}{l}\text { TI } \\
\text { YA } \\
\text { YI } \\
\text { HK } \\
\text { RS }\end{array}$ & $\begin{array}{l}.96 \\
.78 \\
.89 \\
.75 \\
.92\end{array}$ & $\begin{array}{r}-.08 \\
.19 \\
.04 \\
.40 \\
.19\end{array}$ & $\begin{array}{r}7.3 \\
2.1 \\
39.8 \\
237.0\end{array}$ & $\begin{array}{l}.994 \\
.986 \\
.986 \\
.997 \\
.994\end{array}$ & $\begin{array}{r}.79 \\
.87 \\
1.24 \\
.58 \\
.85\end{array}$ & $\begin{array}{l}.998 \\
.999 \\
.997 \\
.998 \\
.996\end{array}$ & $\begin{array}{r}-3.0 \\
-4.0 \\
10.0 \\
5.0 \\
-2.0\end{array}$ & $\begin{array}{l}.44 \\
.43 \\
.63 \\
.56 \\
.41\end{array}$ & $\begin{array}{l}.992 \\
.989 \\
.997 \\
.994 \\
.997\end{array}$ & $\begin{array}{l}15.5 \\
16.0 \\
14.0 \\
14.0 \\
12.0\end{array}$ & $\begin{array}{r}1.14 \\
1.18 \\
.94 \\
.97 \\
.89\end{array}$ & $\begin{array}{r}.996 \\
.999 \\
.999 \\
1.000 \\
.999\end{array}$ \\
\hline $\begin{array}{l}\text { all } \\
*\end{array}$ & $\begin{array}{l}.86 \\
.85\end{array}$ & $\begin{array}{l}.15 \\
.14\end{array}$ & $\begin{array}{r}11.8 \\
8.6\end{array}$ & $\begin{array}{l}.998 \\
.996\end{array}$ & .87 & .999 & & .50 & .995 & 14.3 & 1.02 & 1.000 \\
\hline
\end{tabular}

Values of Parameterş and of Indices of Goodness of Fit in Experiments I to IV 


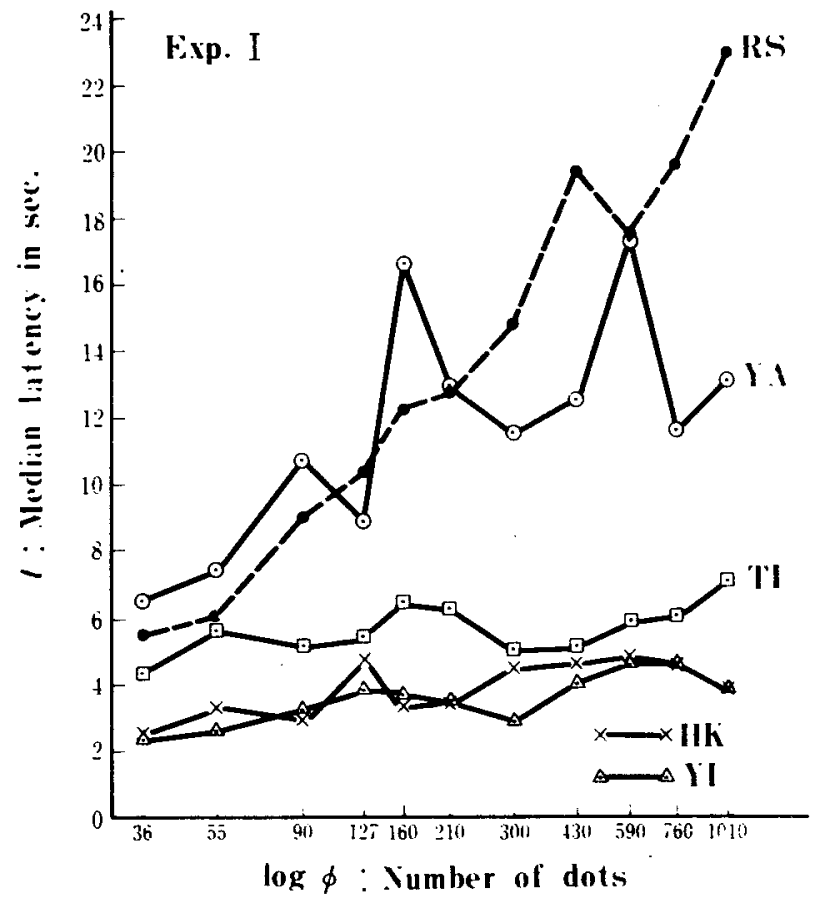

Figure 2. Mean latencies of absolute identification in Experiment I as functions of $\phi$.

the task of the subject was to estimate, in number, the ratio $\mathbf{R}_{\mathbf{j k}}$ between two numerosities. Of each pair, the estimation was repeated twice: once $\phi_{j}$ and $\phi_{\mathrm{k}}$, once $\phi_{\mathrm{k}}$ and $\phi_{\mathrm{j}}$ in spatial arrangement. The stimulus on the left was always taken as unity in defining the ratio $\mathbf{R}_{\mathbf{j k}}$. The same set of values of $\phi_{\mathrm{i}}$ as in Experiment I were used, and comparisons of extremely different stimuli were not included. There seems to be a range within which the subjects can intuitively assess the ratio, and including ratios exceeding this range would contaminate the result. As the matrix of $\mathbf{R}_{\mathbf{j k}}$ was incomplete (see example in Indow \& Ida, 1975), it was necessary to use some special methods (Bock \& Jones, 1968; Gulliksen, 1956; Morrissey; $1955)$ to obtain such values of $\psi_{11}\left(\phi_{i}\right)(i=1,2, \ldots$, 11) that satisfy, in the sense of the least squares, the condition:

$$
\begin{gathered}
\mathbf{R}_{\mathbf{j k}}=\psi_{\mathrm{II}}\left(\phi_{\mathrm{k}}\right) / \psi_{\mathrm{II}}\left(\phi_{\mathrm{j}}\right), \\
\log R_{\mathrm{jk}}=\log \psi_{\mathrm{II}}\left(\phi_{\mathrm{k}}\right)-\log \psi_{\mathrm{II}}\left(\phi_{\mathrm{j}}\right) .
\end{gathered}
$$

In the present case, a modified form of the Gulliken's method was applied for obtaining $\psi_{\text {II }}\left(\phi_{i}\right)$ through Equation 4 (Indow \& Ida, 1975).

II.1. To show the goodness of fit between the two sides of Equation 4, two examples are shown in Figure 3: the most consistent case (H.K.) and the least consistent case (R.S.) in estimating the ratios. The scatter of points around the straight line passing through the origin with the unit slope is given in terms of root mean squares (RMS).
II.2. In accordance with Figure 1, the relation between $\psi_{\mathrm{II}}\left(\phi_{\mathrm{i}}\right)$ and $\phi_{\mathrm{i}}$ was approximately linear on $\log -\log$ coordinates (Figure 4), and Equation 1 was fitted. In the sixth and seventh columns of Table 2 , the values of $\beta$ and of the correlation coefficient $r$ are given. As in I.3., $r$ represents the goodness of fit of Equation 1. Unlike Experiment 1, the unit of $\psi_{\text {II }}$ and hence the origin of $\log \psi_{\mathrm{II}}$ is indeterminate from the present procedure. Hence, the intercept, $\log \alpha$, does not play any essential role. The results of the same subjects as in Figure 3 are given as two individual examples, and one happened to be subject R.S., whose estimation process was different from others' in Experiment I. No such deviation was observed in his verbal reports in Experiment II. The slope, $\beta$, of the pooled data was almost the same in value as $\beta$, in Experiment $I$, in spite of the fact that the correspondence in individual slopes, $\beta$, between Experiments I and II was not very close.

II.3. The latencies in ratio estimation were measured. The medians, $t$ of the respective subjects are plotted in Figure 5 against the ratio $R$. Though slight, an increasing trend of $t$ will be noticed as $R$ deviates from unity to both directions. Individual differences are also noticeable in the overall level of t. The two subjects, Y.A. and R.S., who were slow in making the absolute identification in Experiment $I$ were also slow in making the ratio estimation, compared with the remaining three subjects.

\section{Experiment III: Matching Three Adjacent Intervals}

Because no more than four stimuli could be simultaneously presented, equisecting the whole range of $\phi$ at a time could not go into steps fine enough to define $\psi(\phi)$. Either interpolation or extrapolation has to be repeated successively, and extrapolation was adopted in the present study. In Experiment III, four stimuli, (A, B, C, D), were always presented, with two on either side fixed and the other two adjustable. The task of the subject was to match the three adjacent differences (AB), (BC), and (CD) and to construct a series gradually increasing in numerosity from A to D. Suppose that a standard pair $\left(\phi_{i}, \phi_{i+1}\right)$ was presented in A and B in the beginning, then the number of dots in $C$ and $D$ was adjusted to define $\phi_{i+2}, \phi_{i+3}$. Next, the pair $\left(\phi_{i+2}, \phi_{i+3}\right)$ thus determined was presented in $A$ and $B$, and $\phi_{i+4}$, $\phi_{i+5}$ in $C$ and $D$ was adjusted, and so forth. This ascending procedure was terminated when $\phi$ in $D$ reached the level of 1,000 . Then the first standard pair $\left(\phi_{i}, \phi_{i+1}\right)$ was presented in C and $D$ and the number of dots in $A$ and $B$ was adjusted to define $\left(\phi_{i-2}, \phi_{i-1}\right)$ in A and B. The pair $\left(\phi_{i-2}, \phi_{i-1}\right)$ thus determined was then presented in $C$ and $D$ in the next presentation. This descending procedure was terminated when $\phi$ in $A$ reached the level of 30 . In this way, a series of $\phi_{k}, k=1,2, \ldots, n$ with subjectively 


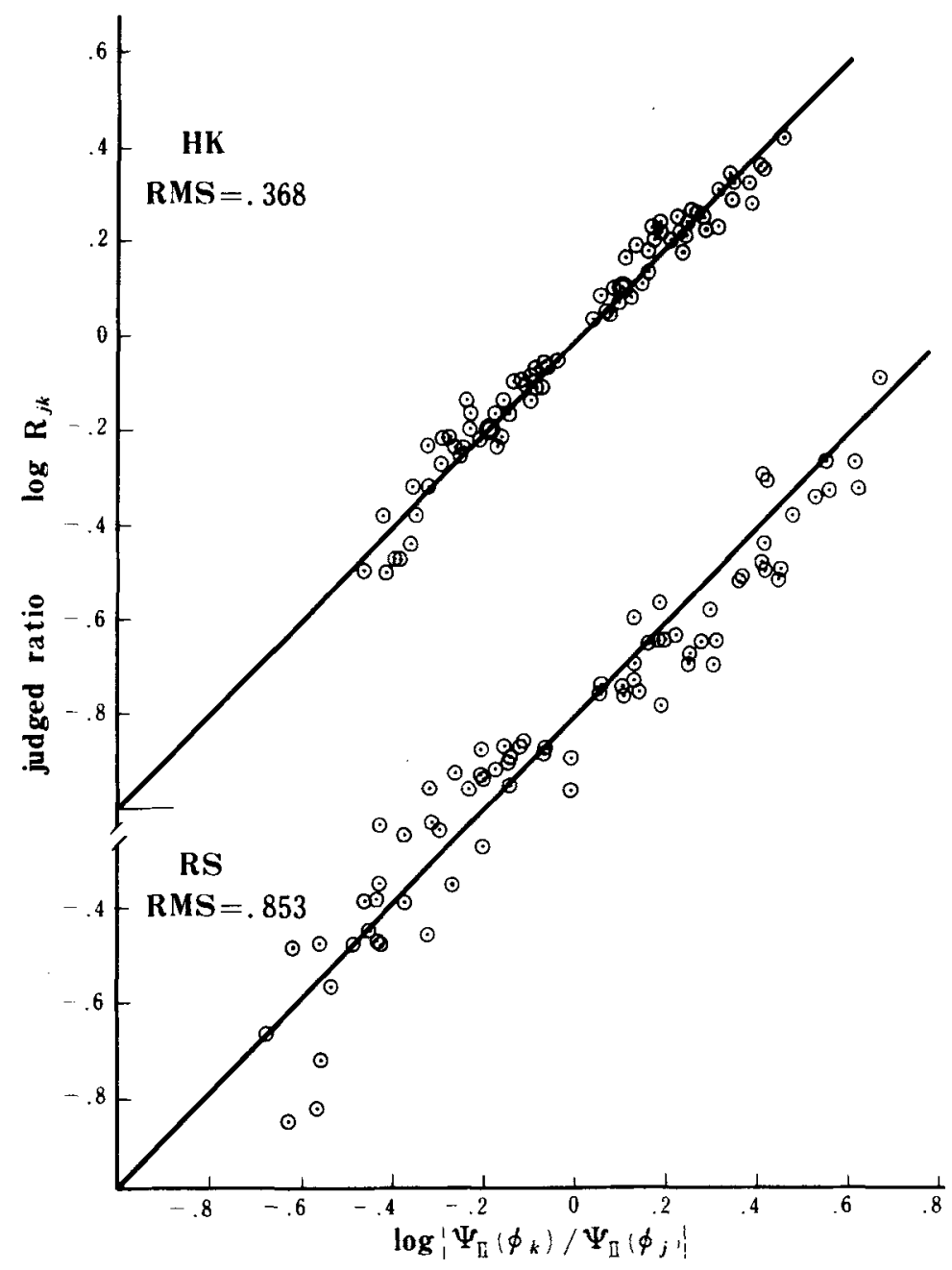

Figure 3. Two examples of consistency of ratio estimations in Experiment II: the most consistent case and the least consistent case.

equal intervals was constructed. The changing of two adjustable stimuli was made by the experimenter as directed by the subject. As each dot pattern consisted of two to four vinyl sheets tightly overlaid, the experimenter adjusted numerosity by changing one or two of the vinyl sheets. During the adjustment, the shutter was closed and the four stimuli were not visible. The adjustment was continued until the subject was satisfied with the uniformity of the gradual change of numerosity of the four in sight. Four stimuli were always arranged so that the numerosity increased from left to right (A to D). The standard pair being presented as the fixed pair in the first presentation defined the size of step in the series $\phi_{k}$, and four series of $\phi_{k}$ were constructed with the respective standard pairs $(127,160),(160,210),(210$, $300)$, and $(300,430)$.

By definition, $\left[\psi_{\mathrm{III}}\left(\phi_{\mathrm{k}+1}\right)-\psi_{\mathrm{III}}\left(\phi_{\mathrm{k}}\right)\right]$ is thought of as a constant in each of the four series. Values of $\phi_{k}$ obtained in each series were plotted against $\psi_{\text {III }}\left(\phi_{k}\right)$, where $\psi_{111}\left(\phi_{k}\right)$ and $\psi_{\text {III }}\left(\phi_{k}+1\right)$ were separated by a constant interval. And then the origin and unit of the scale $\psi_{\text {III }}(\phi)$ were defined in each series so that $\psi_{\mathrm{III}}(100)=20$ and $\psi_{\mathrm{III}}(500)=40$. The definition was arbitrary, of course, except that the interval $(100,500)$ was in the middle of each range of $\phi_{k}$.

III.1. Once converted into the common scale, $\psi_{\text {III }}$, the four series with different standard pairs were in good agreement. Two examples of individual data are shown in the lower part of Figure 6. One is shifted upward, but the unit of ordinate is the same. Each point represents one setting on the common scale. The curve to define $\psi_{\mathrm{III}}(\phi)$ was defined by eye for each subject.

III.2. As a function of $\log \phi, \psi_{\text {III }}(\phi)$ was concave 


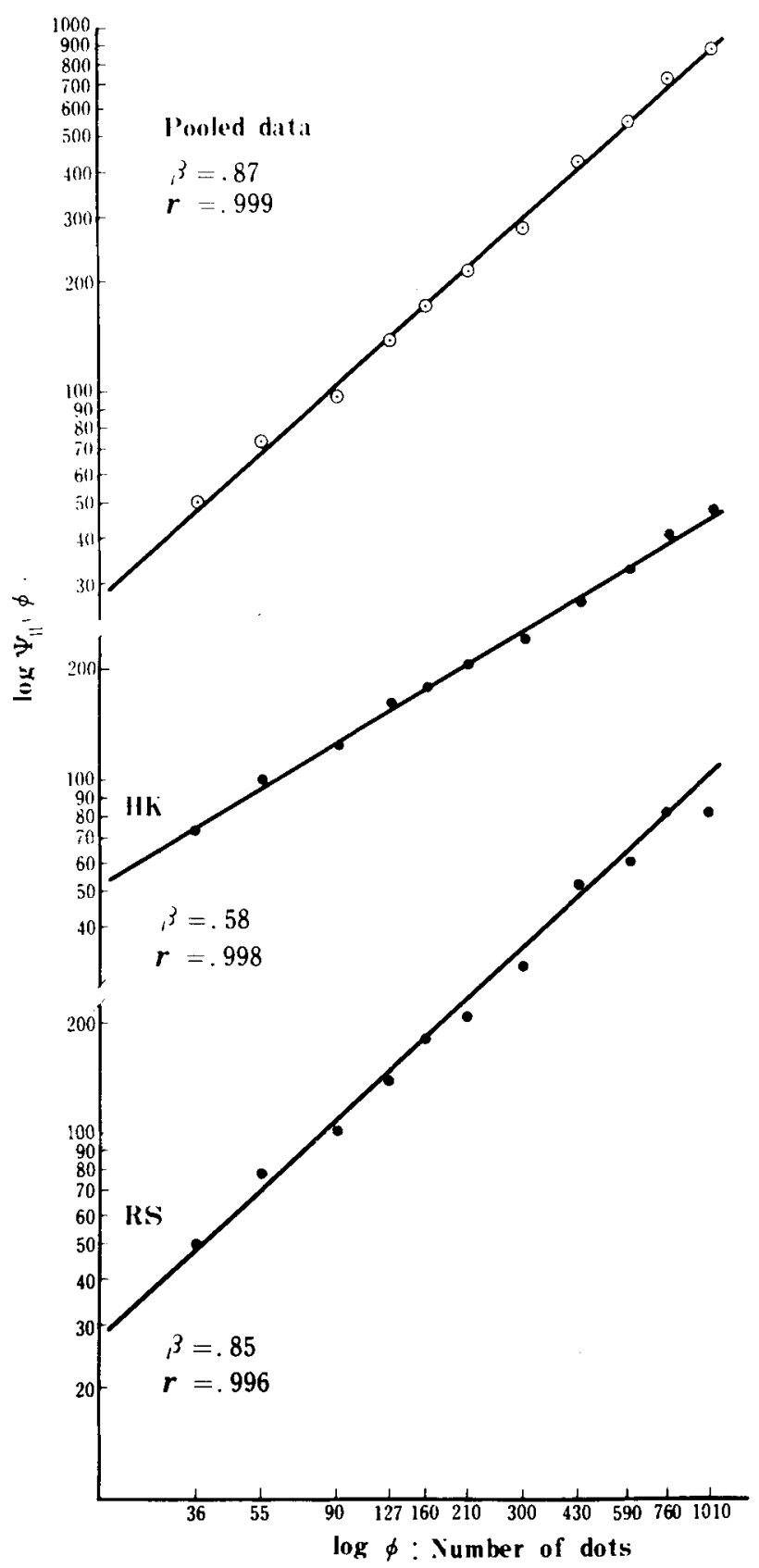

Figure 4. The scales $\psi_{\text {hI }}$ obtained in Experiment II as functions of $\phi$ in log-log coordinates.

upward for each subject. As the origin of $\psi_{111}$ has been arbitrarily defined, such a constant $c$ was sought for by a rule of thumb in the vicinity of $\psi_{\mathrm{III}}(0)$ which makes a new variable

$$
\psi^{\prime}{ }_{\mathrm{III}}(\phi)=\left[\psi_{\mathrm{III}}(\phi)-\mathrm{c}\right]
$$

approximately linear on log-log coordinates. In other words, $\psi^{\prime}$ III $(\phi)$ is a power function of $\phi$ like $\psi_{I}(\phi)$ and $\psi_{\mathrm{II}}(\phi)$. In order to show the linearity, the set of
11 values of $\psi^{\prime}{ }_{\text {III }}\left(\phi_{\mathrm{i}}\right)$ corresponding to the same values of $\phi_{i}$ as used in Experiments I and II were read from the trend $\psi^{\prime}{ }_{\text {III }}(\phi)$ and plotted in log-log coordinates. Two individual cases (the same subjects as in Figure 6) are shown by filled circles in the lower part of Figure 7. Each open circle represents the average of these filled circles of the five subjects. Straight lines were fitted by the method of least squares with equal weights. Values of the constant $c$, the exponent $\beta$, and the correlation coefficient $r$ as an index of the goodness of fit are given in Table 2.

\section{Experiment IV: Summation and Subtraction by a Given Amount}

Three stimuli (A, B, C) were presented at a time and extrapolation to define a series $\psi_{\mathrm{IV}}\left(\phi_{\mathrm{k}}\right), \mathrm{k}=1$, $2, \ldots, n$ was made either by summation or by subtraction. A fixed stimulus $\phi_{0}$ was always presented at the left $(A)$ to define the numerosity to be added to or subtracted from the numerosity at the middle (B) in impression, and $\phi$ at the right (C) was always to be adjusted. The summation procedure started with $\phi_{1}$ in $B$ and $\phi_{2}$ in $C$, and $\phi_{2}$ was adjusted until the numerosity of $\phi_{2}$ appeared to be the sum of the numerosity of $\phi_{1}$ and that of $\phi_{0}$. Then $\phi_{2}$ was presented in $B$ and another stimulus, $\phi_{3}$, in $C$, and $\phi_{3}$ was adjusted so that the numerosity of $\phi_{3}$ appeared to be the sum of the numerosity of $\phi_{2}$ and that of $\phi_{0}$. The subtraction procedure started with $\phi_{n}$ in $B$ and $\phi_{n-1}$ in $C$, and $\phi_{n-1}$ was adjusted so that the numerosity of $\phi_{n}$ appeared to be the numerosity of $\phi_{n}$ subtracted by that of $\phi_{0}$. Then $\phi_{n-1}$ was presented in $B$ and another stimulus, $\phi_{n-2}$, in $C$, and $\phi_{n-2}$ was adjusted. The fixed stimulus in $A$ was either $\phi_{0}=49$ or $\phi_{0}=100$. With the respective values of $\phi_{0}$, two series of stimuli, $\phi_{k}, k=1,2, \ldots, n$, were constructed by summation from $\phi_{1}=30$ and two series by subtraction from $\phi_{n}=1,010$. Each series was terminated when $\phi$ in $C$ exceeded the available stimulus range. As in Experiment III, changing $\phi$ in $C$ was

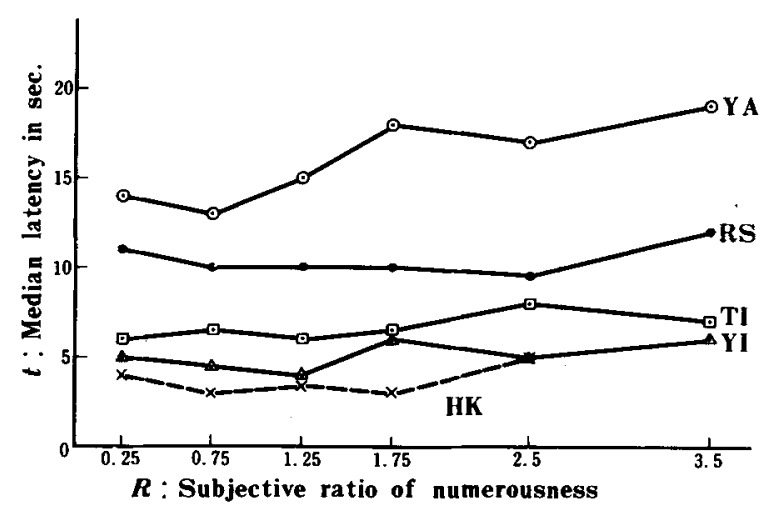

Figure 5. Mean latencies of ratio estimations in Experiment II as functions of ratio $R$. 


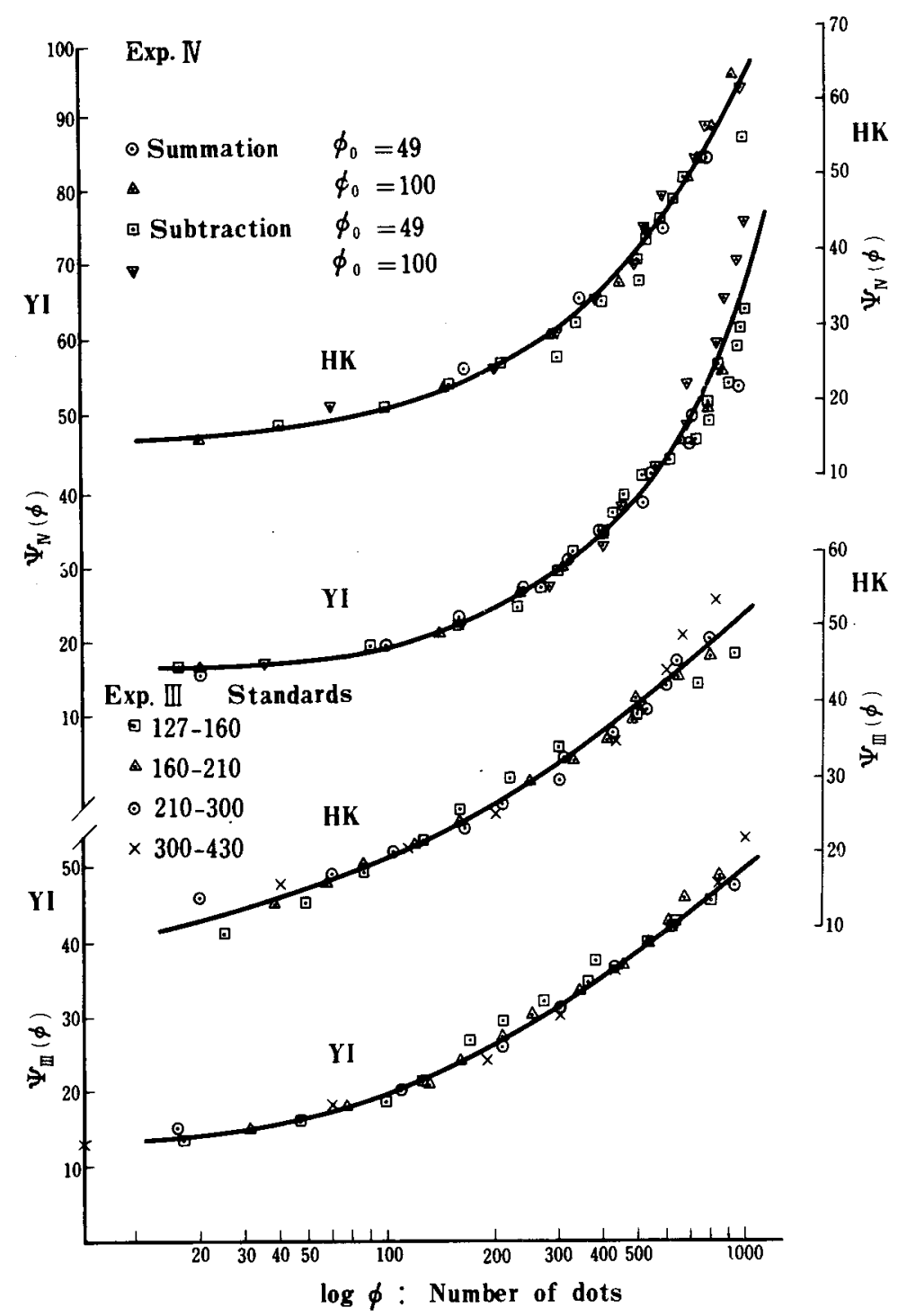

Figure 6. Two examples of individual data in Experiments III and IV each. Each ordinate was arbitrarily defined so that $\psi_{x}(100)=20$ and $\psi_{x}(500)=40$.

made by the experimenter according to the direction of the subject, during which the shutter was closed and the stimuli were not visible. In the summation procedure, the numerosity increased as in Experiment III from left to right (A to $C$ ) and the direction was reversed in the subtraction procedure because $(\phi$ in $B)>$ ( $\phi$ in C). By definition, $\left[\psi_{\mathrm{IV}}\left(\phi_{\mathrm{k}+1}\right)-\psi_{\mathrm{IV}}\left(\phi_{\mathrm{k}}\right)\right]=$ $\psi_{\text {IV }}\left(\phi_{0}\right)$ in each of the four series. As in Experiment III, values of $\phi_{k}$ obtained in each series were plotted once against $\psi_{\mathrm{IV}}\left(\phi_{\mathrm{k}}\right)$, where $\psi_{\mathrm{IV}}\left(\phi_{\mathrm{k}}\right)$ and $\psi_{\mathrm{IV}}\left(\phi_{\mathbf{k}+1}\right)$ were separated with a constant interval. Then the four series were converted into a scale of the common origin and the common unit: $\psi_{\mathrm{IV}}(100)$ $=20$ and $\psi_{\mathrm{IV}}(500)=40$.

IV.1. Once converted, the four series were in good agreement. The curve $\psi_{\mathrm{IV}}(\phi)$ was defined by eye for each subject. Two examples of individual data (the same subjects as in Experiment III) are shown in the upper part of Figure 6 in the same way as in the lower part. Again, each point represents one setting in each series on the common scale.

IV.2. As a function of $\log (\phi), \psi_{\mathrm{IV}}(\phi)$ was concave upward for each subject, like $\psi_{\mathrm{III}}(\phi)$, and the concavity was more marked. By the same procedure as stated in III.2. $\psi_{\mathrm{IV}}(\phi)$ was converted to a power function of $\phi, \psi^{\prime}{ }_{\mathrm{IV}}(\phi)$, and values of the constant $\mathrm{c}$ in Equation 5 used in the conversion are given in Table 2. Plottings corresponding to those in Experiment III are shown in the upper part of Figure 7, and value of the exponent $\beta$ of $\psi^{\prime} \operatorname{IV}(\phi)$ and the index $r$ representing the goodness of fit are also given in Table 2. 


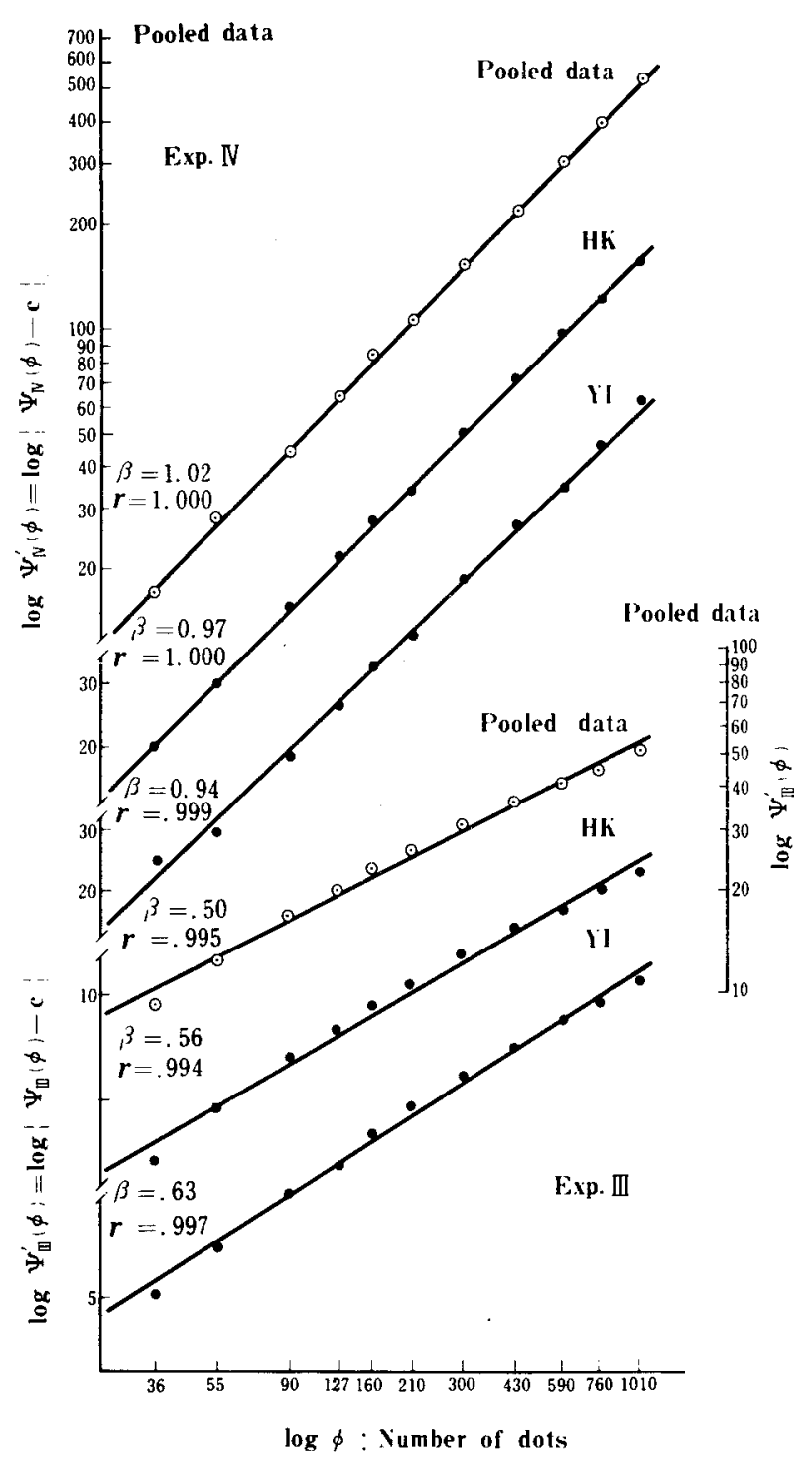

Figure 7. The scales $\psi_{x}^{\prime}=\left(\psi_{x}-\right.$ c) obtained in Experiments III and IV as functions of $\phi$ in $\log -\log$ coordinates.

\section{GENERAL DISCUSSION}

It has been pointed out that adults may use three "quantification operators": subitizing, counting, and estimation (Kaufman, Lord, Reese, \& Valkman, 1949; Klahr, 1973). According to the range of $\phi$ in the present study, the operator under discussion is neither subitizing nor counting. With the range of $\phi, 25$ to 400 , in which the estimation operator takes the leading role, Krueger (1972) showed, by absolute identification and production, that $\psi(\phi)$ was a power function and that there was a striking tendency for subjects to underestimate the number of dots or mark Xs; $\psi(\phi)<\phi$ in that range. The exponents were $0.72,0.78$, and .77 by absolute identification but
0.94 by magnitude production, so that the overall value of $\beta$ was 0.85 . The findings in Experiments I and II of the present article are in agreement with the above mentioned results, though the range of $\phi$ was wider in the present study. Bevan and Turner (1964) reported, under the condition and procedure equivalent to Experiment $I$ (c condition in their study), that $\beta=0.9$ and $\psi(\phi)<\phi$ for $21 \leqslant \phi \leqslant 336$. On the other hand, such cases were also reported with numerosity that $\beta \geqslant 1$ (S. S. Stevens \& Galanter, 1957). In Experiment $I$, such number of dots, $\Phi$, was defined that $\psi(\bar{\phi})=\bar{\phi}$. As $\beta<1$, the number of dots is underestimated, i.e., $\psi(\phi)<\phi$ when $\phi>\bar{\phi}$ and $\psi(\phi)>\phi$ when $\phi<\bar{\phi}$. From Table $2, \bar{\phi}$ is either 11.8 or 8.6 according to whether the data of R.S. are included or not. For simplicity's sake, it was defined that $\bar{\phi}=10$. Interestingly enough, the value of $\bar{\phi}$ corresponds to the points where a discontinuity was observed with the curve relating confidence in rating and $\phi$ (Taves, 1941, according to Figure 1 in Kaufman et al., 1949) as well as with the curve relating reaction time in rating and $\phi$ (Figure 5 in Klahr, 1973). Magnitude estimation data by Kaufman et al. (1949) also exhibited that $\psi(\phi)<\phi$ and $\beta=0.87$ for $\phi>10$ and the result was different for $\phi \leqslant 10$.

In Figure 8, the results of Experiment $I, \psi_{I}$, are plotted against the results of Experiments III and IV prior to the conversion, $\psi_{\text {III }}$ and $\psi_{\text {IV }}$. Filled circles represent two individual results, as in Figures 6 and 7, and open circles represent the average results including all the five subjects. Though not shown, the relationship was exactly the same in the results of subject R.S., whose process of generating $\psi_{\mathrm{I}}$ was somewhat anomalous. The nonlinear relationship between $\psi_{\mathrm{I}}$ and $\psi_{\mathrm{III}}$, the plottings in the lower part, exhibits the typical pattern that is usually found between scales based upon ratio judgments and scales based upon difference judgments (Indow, 1974a, 1974b; S. S. Stevens, 1975). As shown in Table 2, the two scales $\psi_{\mathrm{I}}$ and $\psi_{\mathrm{II}}$ are almost identical on the average, which suggests that the subject assigned, in Experiment I, numbers proportional to the impression of numerosity. The class of scale in which this kind of proportionality holds is called magnitude scale, and let us denote by $\psi_{\mathrm{M}}$ the power function with $\beta=0.86$. The procedure of Experiment III is essentially the same with the conventional method of equisection, and let us denote $\psi_{\text {III }}$ by $\psi_{\mathrm{D}}$, because matching adjacent differences in impression plays the fundamental role. It is well known that $\psi_{\mathrm{M}}$ and $\psi_{\mathrm{D}}$ are not linearly related in general and $\psi_{M}$ is a positively accelerated function of $\psi_{\mathrm{D}}$. In the lower part of Figure 9, the relationship between $\psi_{M}$ and $\psi_{D}$ is shown with three sense modalities: two scales of whiteness, Munsell value $V$ as $\psi_{\mathrm{M}}$ (Munsell, Sloan, \& Godlove, 1933) and bril as $\psi_{M}$ (S. S. Stevens \& J. C. Stevens, Note 2), two 


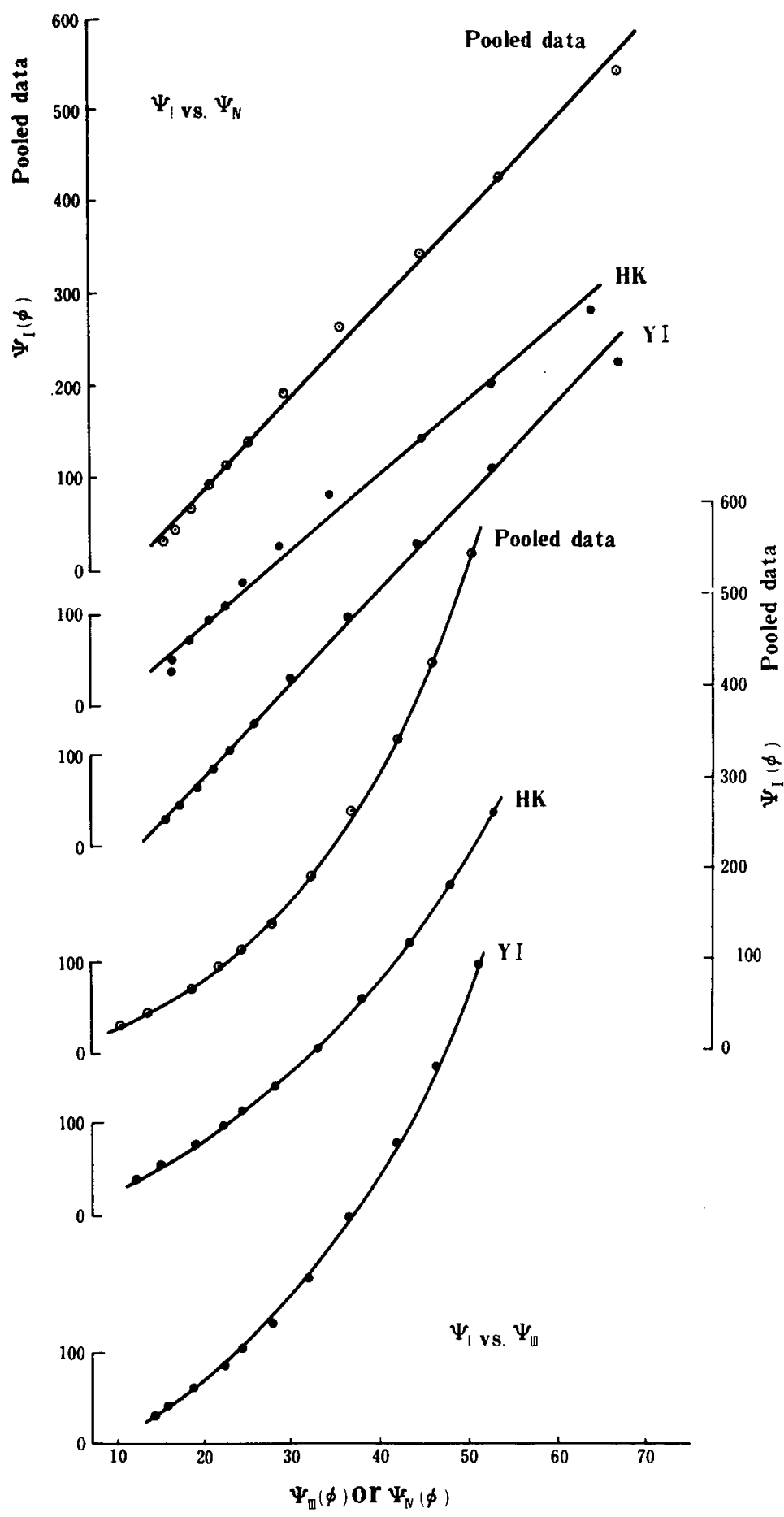

Figure 8. Relation between $\psi_{1}$ by the absolute identification and $\psi_{\text {III }}$ or $\psi_{\text {IV }}$, both based upon difference judgments: two examples of individual data and the representative data each.

scales of loudness, $\lambda$ as $\psi_{\mathrm{D}}$ (Garner, 1954) and sone as $\psi_{\mathrm{M}}(\mathrm{S}$. S. Stevens, 1955), and two scales of sweetness, $\tau$ as $\psi_{\mathrm{D}}$ (Indow, 1966, 1969) and gust as $\psi_{\mathrm{M}}$ (Beebe-Center, 1949). In the respective cases, $\psi_{M}$ and $\psi_{\mathrm{D}}$ were obtained in different experiments. The smallest and largest values of $\psi$ definable on the widest range of $\phi$ common to both experiments are plotted in Figure 9 at the points marked by triangles in the abscissa as well as on the ordinate on the right. When superimposed by equating the respective 


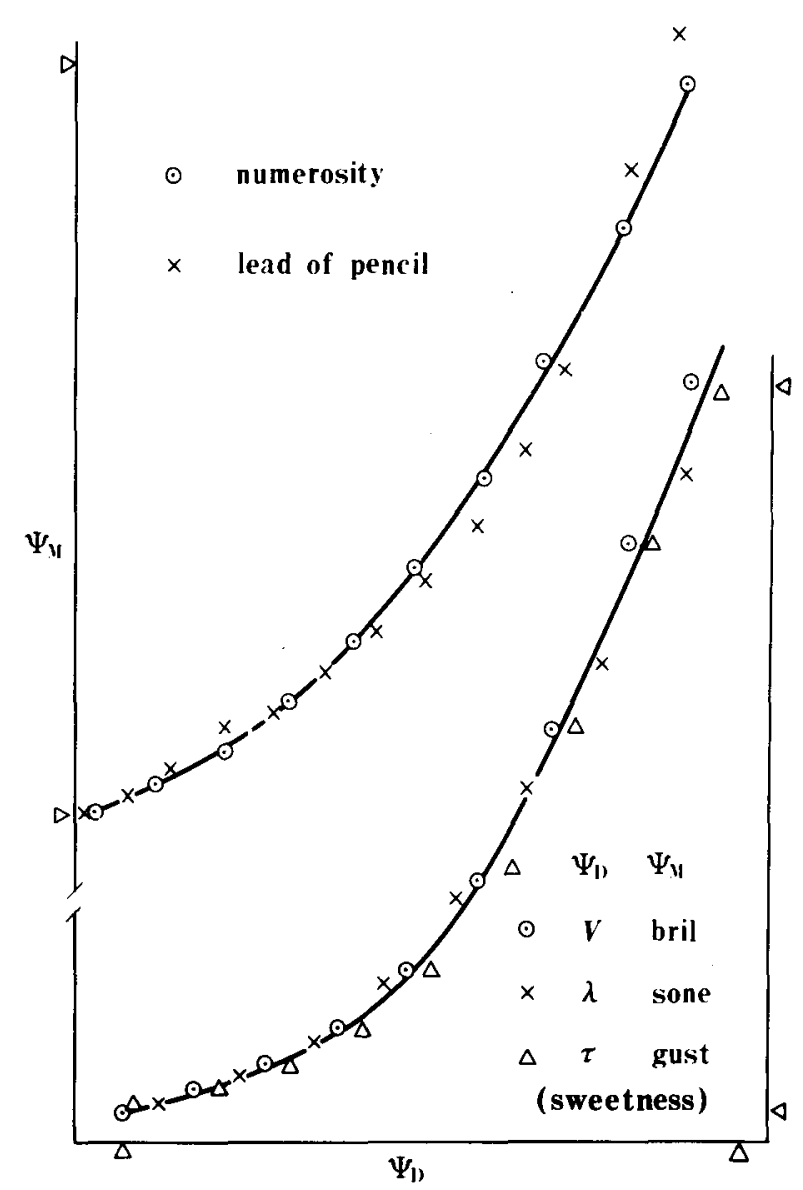

Figure 9. Examples of nonlinearity between magnitude scales $\psi_{M}$ and difference scales $\psi_{D}$.

ranges of $\psi$ in this way, the agreement among the three sets is impressive. Notice that each of these three sets of data is based upon the widest possible range of $\phi$ for the modality. The agreement seems to suggest that the nonlinearity between $\psi_{\mathrm{M}}$ and $\psi_{\mathrm{D}}$ has its origin in the process through which the quantitative judgment is generated rather than in the preceding sensory processes themselves. In the upper part of Figure 9, the relation between $\psi_{M}$ and $\psi_{D}$ for subjective hardness of pencil lead (Indow, 1957) is shown by circles. As the pairs of triangles in the ordinate on the left have the same interval as the pairs in the ordinate on the right, the respective ranges of $\psi_{\mathrm{M}}$ and $\psi_{\mathrm{D}}$ of the upper plottings are the same with those of the lower plottings. Again, the nonlinearity concave upward is observed of the results with pencil lead but slightly less curved than the plottings in the lower part. Evidently, the curvature is affected by the range of stimulus $\phi$ over which $\psi_{\mathrm{M}}(\phi)$ and $\psi_{\mathrm{D}}(\phi)$ are defined. It cannot be claimed that pencil lead covers the widest possible range for the impression of hardness. As the same will be true for the range of $\phi$ in the present experiment, 30 to 1,010 in the area of $19.5 \times 24.5 \mathrm{~cm}$, the average results of $\psi_{\mathrm{I}}$ and $\psi_{\text {III }}$ were superimposed upon the results with pencil lead by equating the respective ranges so as to yield the closest fit. Interestingly enough, the fit of the order as shown in Figure 9 was obtained.

The plottings in the upper part of Figure 8 show that $\psi_{\mathrm{I}}$ is approximately linear to $\psi_{\mathrm{IV}}$, though convexity of a slight degree is noticeable. In other words, the relation between $\psi_{I}$ and $\psi_{\text {IV }}$ is entirely different from that between $\psi_{\mathrm{I}}$ and $\psi_{\mathrm{III}}$. Notwithstanding the fact that the two scales, $\psi_{\text {III }}$ and $\psi_{\text {IV }}$, have been constructed by the equisection procedure in a broader sense, $\psi_{\text {IV }}$ behaves differently from $\psi_{\text {III }}$, and that fact is also apparent in Figures 6 and 7. The difference between Experiments III and IV lies in the way of defining intervals of constant size. As shown in Figure 10, Experiment III gave such a series of stimuli, $\phi_{i}$, in which hatched segments match with each other (A), whereas in Experiment IV $\left[\psi\left(\phi_{i+1}\right)-\psi\left(\phi_{i}\right)\right]$, etc., were always matched with $\psi\left(\phi_{0}\right)(\mathrm{B})$, and $\psi\left(\phi_{0}\right)$ is not an interval between two levels of $\psi$ but an absolute amount starting from the zero level, so to speak. The operation of intuitive summation or subtraction (Experiment IV) will not be feasible in most sense modalities, and apparent length of line (Kreuger, 1970), duration in time, and numerosity are exceptional in the sense that the subject is capable of performing Task B with the same ease or difficulty as in the Task A. Logically, Procedure B can be regarded as a special case of Procedure A. Psychologically, however, the two procedures seem to be quite different in nature. According to S. S. Stevens (1975), the plottings in the lower part of Figure 8 imply that numerosity is a prothetic continuum and, in Experiments $I$ and II, the exponent $\beta$ was about 0.86 . It will be of interest that such an operation concerning difference judgments
(A)

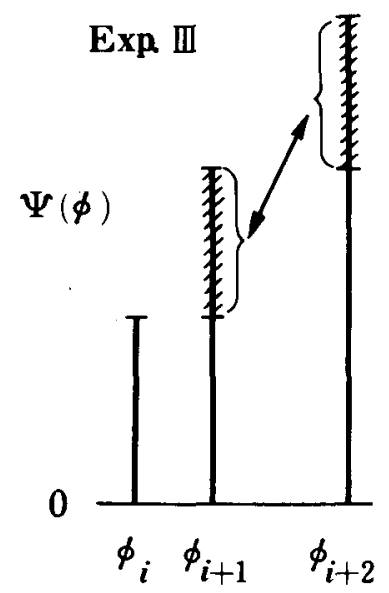

(B)

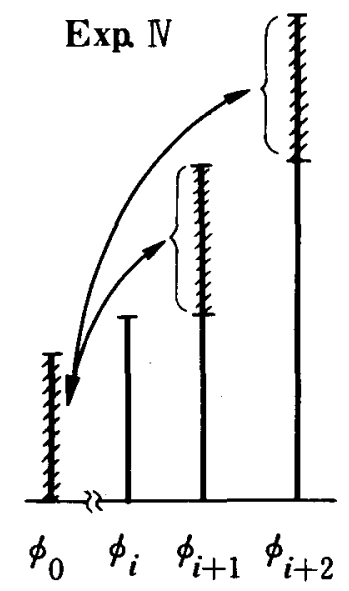

Figure 10. Schematic illustrations of the procedures in Experiment III and in Experiment IV. 
(Experiment IV) has been found for one modality, numerosity, one which at least yields an approximately linear relation with $\psi_{\mathrm{I}}$ which is based upon ratio judgments and has the exponent differing from unity. In the case of apparent length of line or duration of time, the relation between $\psi_{\mathrm{D}}$ and $\psi_{\mathrm{M}}$ would be also approximately linear, but the exponent for $\psi_{\mathrm{M}}$ is very close to unity.

An attempt was made in Figure 11 to plot the four scales $\psi_{\mathrm{x}}(\phi)$ in the present experiments on a common scale. As the scale $\psi_{\mathrm{I}}(\phi)$ is absolute in the sense discussed before, the remaining scales were converted into this scale in the following way. The fact that $\psi_{\mathrm{I}}(\bar{\phi})=\bar{\phi}$ and $\bar{\phi}=10$ was utilized in the conversion. When the scale of $\psi_{\mathrm{II}}(\phi)$ is adjusted so that $\psi_{\mathrm{II}}(10)$ $=10, \psi_{\mathrm{II}}(\phi)$ and $\psi_{\mathrm{I}}(\phi)$ are indistinguishable because both are power functions with the exponents $\beta$ of about the same value. Both the scales $\psi_{\text {III }}$ and $\psi_{I V}$ are of the form of power function starting from a point $\mathrm{c}(\neq 0)$ :

$$
\psi(\phi)=\alpha \phi^{\beta}+\mathrm{c},
$$

and the scale of ordinate was adjusted so that $\psi(10)$

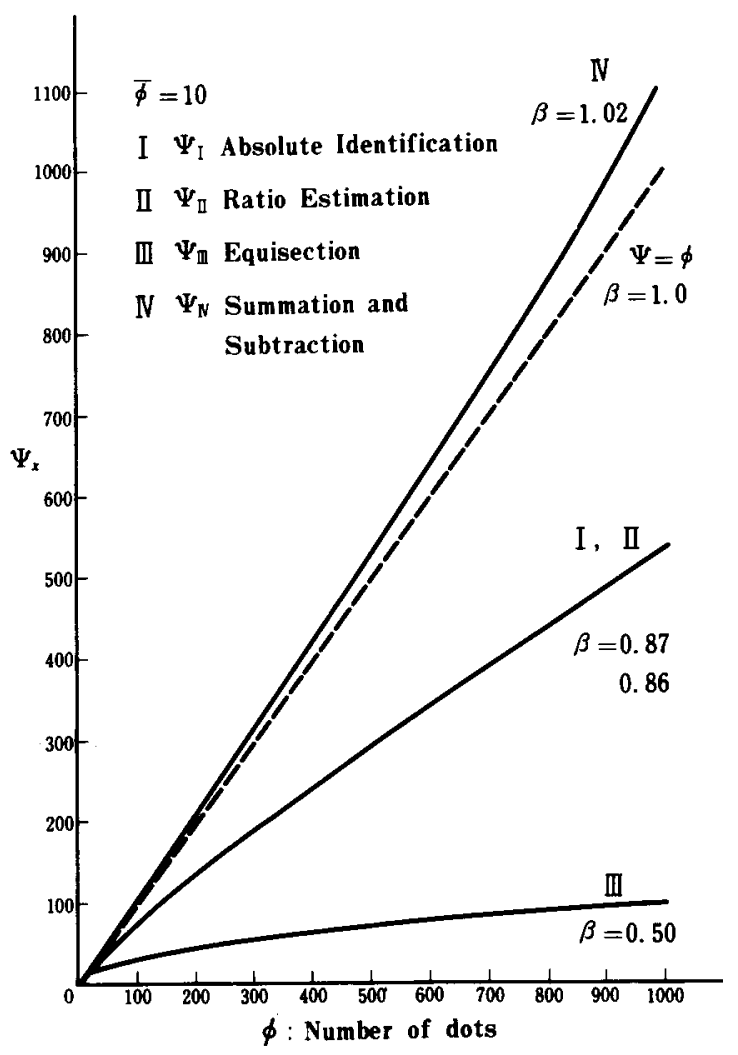

Figure 11. Four scales obtained in Experiments I and IV plotted in linear coordinates. At $\phi=10$, all scales take the value of 10 .
$=10$. Notice that the value of $\mathrm{c}$ is also affected, but nothing else is changed by the adjustment. The scales $\psi_{\mathrm{III}}(\phi)$ and $\psi_{\mathrm{IV}}(\phi)$ thus defined are given in Figure 11 . One will be surprised by seeing how large is the difference between $\psi_{\mathrm{III}}(\phi)$ and $\psi_{\mathrm{IV}}(\phi)$ in linear coordinates. The difference between the curve I, II and the curve III is responsible for the relation being concave upward in the lower plottings in Figure 8, and the difference between the curve I, II and the curve IV is reflected in the upper plottings in Figure 8 primarily into the slope of the straight line and secondarily into the slight convexity of points.

\section{REFERENCE NOTES}

1. Stevens, S. S. Assessment of noise: Calculation procedure mark VII. Laboratory of Psychophysics, Harvard University, 1969, PPR-355-128.

- 2. Stevens, S. S., \& Stevens, J. C. The dynamics of visual brightness. Harvard University, Psychophysical Project Report, 1960, PPR.246.

\section{REFERENCES}

Beebe-Center. J. G. Standards for use of the gust scale. Journal of Psychology, 1949. 28, 411-419.

Bevan, W., \& Turner, E. D. Assimilation and contrast in the estimation of number. Journal of Experimental Psychology, $1964,67,458-462$.

Bock. R. D., \& JoNes. L. V. The measurement and prediction of judgment and choice. San Francisco: Holden-Day, 1968.

GARNER, W. R. A technique and a scale for loudness measurement. Journal of the Acoustic Society of America, 1954, 26. 73-88.

Gulliksen, H. A least squares solution for paired comparisons with incomplete data. Psychometrika, 1956, 21, 125-134.

Hopkinson, R. G. Assessment of brightness. Illuminating Engineering, 1957, 52, 211-222.

InDow, T. Scaling of hardness of lead of pencil. Hinshitsu Kanri. 1957. 97-100 (in Japanese).

InDow, T. A general equi-distance scale of the four qualities of taste. Japanese Psychological Research, 1966, 8, 136-150.

InDow. T. An application of the $\boldsymbol{\tau}$ scale of taste: Interaction among the four qualities of taste. Perception \& Psychophysics, $1969,5,347-351$.

InDow. T. Colour atlases and colour scaling. Association Internationale de la Couleur, Colour 73. London: Adam Hilger. 1974. Pp. 137-152. (a)

InDow, $\mathrm{T}$. Scaling of saturation and hue shift: Summary of results and implications. In H. R. Moskowitz, B. Scharf, \& J. C. Stevens (Eds.). Sensation and measurement-Papers in honor of S. S. Stevens. Boston: Reidel, 1974. Pp. 351-362. (b)

INDow, T., \& IDA, M. On scaling from incomplete paired comparison matrix. Japanese Psychological Research, 1975, 17, 98-105.

Kaufman, E. L., Lord, M. W., Reese, T. W., \& Volkmann, J. The discrimination of visual number. American Journal of Psychology, 1949, 62, 498-525.

KLAHR, D. Quantification processes. In W. Chase (Ed.). Visual information processing. New York: Academic Press, 1973. Pp. 3-34.

KruEger, L. E. Apparent combined length of two-line and four-line sets. Perception \& Psychophysics, 1970. 8. 210-214.

Krueger. L. E. Perceived numerosity. Perception \& Psychophysics, 1972, 11, 5-9.

ManN, L. The social psychology of waiting lines. American Scientist, 1970, 58, 390-398. 
MorRissey, J. H. New method for the assignment of psychometric scale values from incomplete paired comparisons. Journal of the Optical Society of America, 1955, 45, 373-378.

Munsell, A. E. O., Sloan, L. L., \& Godlove, 1. H. Neutral value scales. 1. Munsell neutral value scale. Joumal of the Optical Society of America, 1933, 23, 394-418.

Stevens, J. C., \& Stevens, S. S. Brightness function: Effects of adaptation. Journal of the Optical Society of America, 1963, 53, 375-385.

Stevens, S. S. On the problem of scales for the measurement of psychological magnitudes. Journal of Unified Science, $1939,9,94-99$.

SteVens, S. S. The measurement of loudness. Journal of the
Acoustical Society of America, 1955, 27, 815-829.

Stevens. S. S. (G. Stevens, Ed.) Psychophysics: Introduction to its perceptual, neural, and social prospects. New York: Wiley, 1975.

Stevens, S. S., \& Galanter, E. H. Ratio scales and category scales for dozen perceptual continua. Journal of Experimental Psychology, 1957, 54, 377.411.

TAves, E. M. Two mechanisms for the perception of visual numerousness. Archives of Psychology, New York, No. 265, 1941.

(Received for publication December 7, 1976; revision received May 13, 1977.) 